\title{
Modification, Degradation, and Stability of Polymeric Surfaces Treated with Reactive Plasmas
}

\author{
CHRISTOPHER M. WEIKART, HIROTSUGU K. YASUDA \\ Center for Surface Science and Plasma Technology, Department of Chemical Engineering, University of \\ Missouri-Columbia, Columbia, Missouri, 65211
}

Received 15 February 2000; accepted 8 June 2000

\begin{abstract}
The degradation, modification, and stability of polymeric surfaces exposed to chemically reactive $\mathrm{O}_{2}$ and $\mathrm{H}_{2} \mathrm{O}$-vapor plasmas were investigated. Specifically, the effects of these plasmas on etching rate, surface morphology, wetting instability, and fluid-holding capability were studied. Wetting instability is reflected by hydrophobic recovery and can be examined by the Wilhelmy balance method. Although hydrophobic recovery is usually attributed to surface configuration change, there are actually two types: reversible and permanent. Reversible hydrophobic recovery is caused by surface configuration change, whereas permanent hydrophobic recovery is caused by the creation of oxidized surface oligomers. This study distinguishes the two by identifying differences in the shapes of the corresponding Wilhelmy force loops and in the fluidholding parameter. The presence of surface oligomers was most detrimental to wetting stability and fluid-holding capability but could be controlled via the type of reactive gas, the discharge conditions, and the polymer substrate. In general, polymers most susceptible to $\mathrm{O}_{2}$-plasma etching had the least surface oligomers and vice versa, whereas $\mathrm{H}_{2} \mathrm{O}$-vapor plasma suppressed surface oligomers on polymers less susceptible to etching. (C) 2000 John Wiley \& Sons, Inc. J Polym Sci A: Polym Chem 38: 3028-3042, 2000
\end{abstract}

Keywords: fluid-holding capability; surface oligomers; reactive plasmas; surface configuration change; Wilhelmy; hydrophobic recovery

\section{INTRODUCTION}

Chemically reactive plasmas have been used extensively in the fields of semiconductor devices and polymer surface modification for enhanced adhesion, improved printability, and biocompatibility. Plasmas are employed in the manufacturing of silicon chips to modify the surface of silicon wafers in the micrometer to submicrometer range via etching and doping techniques. However, the field of surface modification is primarily concerned with the outermost surface region on the molecular level (i.e., angstroms). This surface region, typically called the surface state, is particu-

Correspondence to: H. K. Yasuda

Journal of Polymer Science: Part A: Polymer Chemistry, Vol. 38, 3028-3042 (2000) (ㅇ) 2000 John Wiley \& Sons, Inc. larly important because it interacts with the working environment to fulfill a specific interfacial function.

The term surface state is used to indicate a region of the surface at an interface that has considerably different properties than the same material in bulk. ${ }^{1}$ For example, the surface state of water, that is, vicinal water at water/solid interfaces, exhibits structural and physical properties markedly different from those of the bulk fluid. ${ }^{2}$ Similarly, the surface state of polymeric materials possesses far greater mobility than the bulk macromolecules. This enhanced mobility is achieved through the rotation of functional groups about the polymer chain backbone rather than the long-chain segmental motion of macromolecules. The specific spatial arrangement of functional groups interfaced with a contacting 
medium is called the surface configuration. This is not to be confused with configurations of macromolecules. For example, the configuration of atoms in a macromolecule of gelatin gel is known to contain hydrophilic groups; however, gelatin gels are surprisingly hydrophobic. ${ }^{3}$ This is because all the hydrophilic groups at the gelatin/air interface are oriented toward the bulk. Thus, it is not the configuration of hydrophilic groups with respect to the polymer backbone but rather the surface configuration of those groups that dictates wettability. The reorientation of surface-state functional groups in response to a change in the interfacing environment is called surface configuration change by $\mathrm{H}$. Yasuda et al. ${ }^{1,4-7}$

The stability of the surface state after plasma modification is a major concern for any investigator attempting to improve wettability by plasma surface modification. Hydrophobic recovery, that is, the decrease in surface hydrophilicity, as indicated by an increase in contact angle with aging time, is a common example of surface instability encountered after plasma modification with reactive plasmas. ${ }^{4,8,9}$ More often than not, hydrophobic recovery is a result of surface configuration change. However, certain polymers exposed to reactive plasmas, that is, impinging free radicals, ions, and UV light and radiation, are more susceptible to degradation than modification. When exposed to radiation during plasma treatment, some polymers, such as poly(methyl methacrylate) (PMMA) and polytetrafluoroethylene, are of the degrading type, whereas others, such as polyethylene and silicone elastomers, are of the crosslinking type. ${ }^{10}$ Plasma-induced degradation in polymers is detrimental to wetting stability because, as this article reports, it can create instabilities in the form of oxidized oligomers.

Reactive plasmas vary in their ability to chemically etch polymers ${ }^{11-14}$ depending on the volatility of the products they form. The formation of nonvolatile oligomers may dominate etching, depending on the type of substrate, the reactive gas used, and the discharge conditions. Oxidized oligomers resting on a surface provide an extremely unstable surface state because they are not permanently attached. For example, Inagaki et al. ${ }^{15}$ reported that the hydrophobic recovery of $\mathrm{O}_{2}$ plasma-treated polyetheretherketone (PEEK) films after washing with acetone was due to degradation products, that is, surface oligomers. Furthermore, exposure to remote $\mathrm{O}_{2}$ plasma rather than direct $\mathrm{O}_{2}$ plasma was more effective in minimizing hydrophobic recovery. Poncin-Epaillard et al. ${ }^{16}$ used ${ }^{1} \mathrm{H}$ NMR to show that oxidized oligomers with a molecular weight of 500 were generated on the surface of plasma polymer exposed to $\mathrm{CO}_{2}$ plasma. These oxidized oligomers were faulted for the increase in contact angle after washing with water.

In terms of surface sensitivity, contact-angle measurement is superior to other more complex analytical techniques. Contact-angle measurement is a nondestructive technique that probes the outermost portion (i.e., a few angstroms) of the surface that most intimately contacts the working environment. Dynamic contact-angle measurement by the Wilhelmy balance method can yield important information regarding the perturbability or instability of the surface to changing environments that may result from surface modification. Proper interpretation of Wilhelmy force-loop data is necessary to understand such surface instability phenomena.

The objective of this study was to distinguish between the two causes of wetting instability on conventional polymers exposed to reactive plasmas, that is, hydrophobic recovery due to the production of oxidized surface oligomers and hydrophobic recovery due to surface configuration change. The sensitivity of various polymers to reactive plasmas was examined by differences in etching rate, surface morphology, wetting stability, and fluid-holding capability.

\section{EXPERIMENT}

\section{Materials}

Sheets of 1-mm-thick low-density polyethylene (LDPE), polyoxymethylene (POM), PMMA, poly(ethylene terephthalate) (PET), and silicon rubber (SR) were purchased from Goodfellow Corp. and cut into $20 \times 25 \mathrm{~mm}$ plates. The plates were ultrasonically washed in a 5\% solution of soap water for $30 \mathrm{~min}$. They were then thoroughly rinsed with distilled, deionized (DDI) water, allowed to dry in ambient air for $24 \mathrm{~h}$, and then placed in a dry desicator.

The gases used to generate reactive plasmas included the following: oxygen (99.9\% minimum) supplied by Scott Specialty Gases, Inc. and water vapor from an in-house DDI water supply.

DDI water was produced in-house: the water was first distilled and then passed once through an ion exchange column. The resistivity of the DDI water was $40 \mathrm{M} \Omega \mathrm{cm}$. DDI water was used 
for plasma modification and Wilhelmy balance measurements.

\section{Wilhelmy Balance Method}

The Wilhelmy balance apparatus was composed of a Sigma 70 (KSV Instruments, Ltd., Finland) automatic tensiometer interfaced with a personal computer. The tensiometer measures the force exerted by water on a partially immersed thin plate with a measuring range of $25 \mathrm{mN}$ and a resolution of $1 \mu \mathrm{N}$. The total force exerted on a sample when it touches the surface of the water is given by the following force balance equation:

$$
F_{\text {Total }}=M g-\rho g t H d=L \gamma_{L} \cos \theta
$$

where $F_{\text {Total }}$ is the total force exerted on the sample, $M$ is the mass of the plate, $g$ is the gravitational acceleration, $\rho$ is the liquid water density, $t$ is the thickness of the plate, $H$ is the width of the plate, $d$ is the immersion/emersion depth, $L$ is the plate perimeter $[L$ equals; $2 \times$ (thickness + width)], $\gamma_{L}$ is the liquid water surface tension, and $\theta$ is the contact angle at the liquid/solid/air contact line. Because the tensiometer is automatically zeroed when a sample plate touches the surface of the water, the gravitational force of the sample may be neglected. Therefore, the actual force measured by the tensiometer is given by the following equation:

$$
F=L \gamma_{L} \theta-\rho g t H d
$$

The measured force, $F$ in eq 2 , is the difference between the interfacial force between the water and sample plate (wetting force) and the buoyant force from the immersed portion of the plate. The total force, $F$, is divided by the sample plate perimeter, $L$, to give force per unit length, $F / L(\mathrm{mN} /$ $\mathrm{m}$ ), or the abscissa of force-loop plots.

Advancing contact angles from the first immersion, $\theta_{D, a, 1}$, and second immersion, $\theta_{D, a, 2}$, were calculated by the extrapolation of the first and second immersion lines, $(F / L)_{D, a, 1}$ and $(F / L)_{D, a, 2}$, respectively, to zero immersion depth and the use of eq 2.

Fluid-holding time (FHT) was calculated by the division of the immersion velocity $(\mathrm{mm} / \mathrm{min})$ by the length of the region where the second immersion line retraces the first emersion line measured from a Wilhelmy force loop. However, FHT cannot be used in an absolute sense because it is dependent on Wilhelmy experimental conditions. Therefore, immersion and emersion velocity was kept constant at $5 \mathrm{~mm} / \mathrm{min}$ without the motion of the substrate being halted at any time during each wetting cycle. Details of the FHT parameter and its relationship to aqueous film stability can be found elsewhere. ${ }^{17}$

\section{Plasma Reactor System}

Reactive plasmas were generated in a bell-jar reaction chamber. In this reactor setup, a $15-\mathrm{kHz}-$ audio-frequency PE-1000 generator (Advanced Energy Industries, Inc.) powers a pair of titanium electrodes $(18.1 \times 18.1 \times 0.16 \mathrm{~cm})$ separated by a distance of $10 \mathrm{~cm}$. A magnetron system is mounted on the back of each electrode. The magnetron confines the glow discharge to the interelectrode space. Continuous movement of a sample into and out of the glow discharge combined with magnetron enhancement improves the uniformity and reproducibility of the plasma treatment. Therefore, each sample was allowed to spend about $20 \%$ of the total discharge time rotating into and out of the glow discharge on a wheel; this is called plasma exposure time throughout the article. Water vapor was fed from a reservoir of liquid water that was cooled in an ice bath to $0{ }^{\circ} \mathrm{C}$. The flow rate is controlled by a manual needle valve. The valve and tubing leading into the reactor are heated with electrical heating tape. Further details of the reactor setup can be found elsewhere. ${ }^{18}$

\section{Etching Rates}

Etching rates were determined gravimetrically by the weighing of the polymer plates with a Mettler AT261 Delta Range scale before and after exposure to $\mathrm{O}_{2}$ and $\mathrm{H}_{2} \mathrm{O}$-vapor plasmas. The plates were weighed within a measuring range of 0.01 mg. The difference in weight before and after exposure to plasma was normalized to the surface area of the plates, which was estimated to be $10.74 \mathrm{~cm}^{2}$ on the basis of the plate dimensions, 20 $\times 25 \times 1 \mathrm{~mm}$.

\section{Scanning Electron Microscopy (SEM)}

SEM micrographs were taken with an Amray Model 1600 Turbo at $20 \mathrm{keV}$ and 20,000× magnification. Samples were sputter-coated with $15 \mathrm{~nm}$ of $\mathrm{Au}$. 

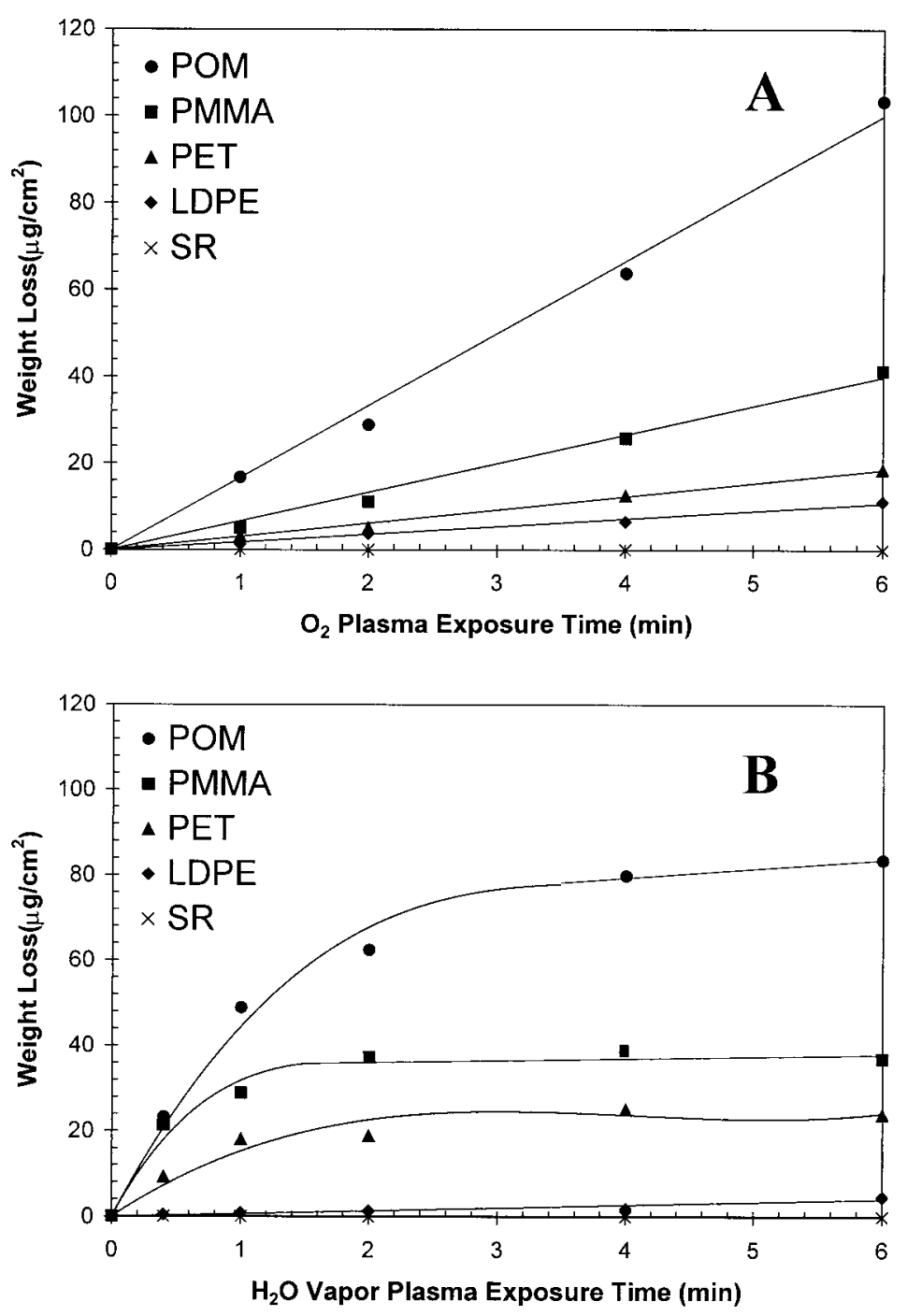

Figure 1. Normalized weight loss of five conventional polymers as a function of exposure time to (A) oxygen plasma and (B) water-vapor plasma. Plasma discharge conditions were fixed for both plasmas at $1 \mathrm{sccm}, 50 \mathrm{mTorr}$, and $36 \mathrm{~W}$.

\section{RESULTS AND DISCUSSION}

\section{Chemical Etching and Morphology}

Chemically reactive plasmas are formed by inorganic and organic gases such as $\mathrm{H}_{2}, \mathrm{O}_{2}, \mathrm{CO}_{2}, \mathrm{H}_{2} \mathrm{O}$ (vapor), $\mathrm{N}_{2}$, and $\mathrm{CF}_{4}$. Unlike polymer-forming plasmas, reactive plasmas do not form solid polymeric deposits but physically and chemically react with the surface of polymeric materials. These reactions can result in crosslinking, oxidation, or etching of the surface macromolecules. However, the chemical reaction of reactive plasmas with the surface of polymeric materials, called chemical etching, is typically the dominant process. Chem- ical etching is an ablative process that causes oxidation and subsequent chain scission of the outermost macromolecules. This ultimately removes surface material in the form of volatile organic products that exit the reactor via the vacuum system.

The chemical structure of macromolecules and the type of reactive gas determine the extent of degradation and thus the amount of material removed, that is, the weight loss. ${ }^{11-14}$ The extent of degradation caused by oxygen plasmas varies according to the type and arrangement of atoms in the polymer. For example, polymers composed of oxygen-containing 

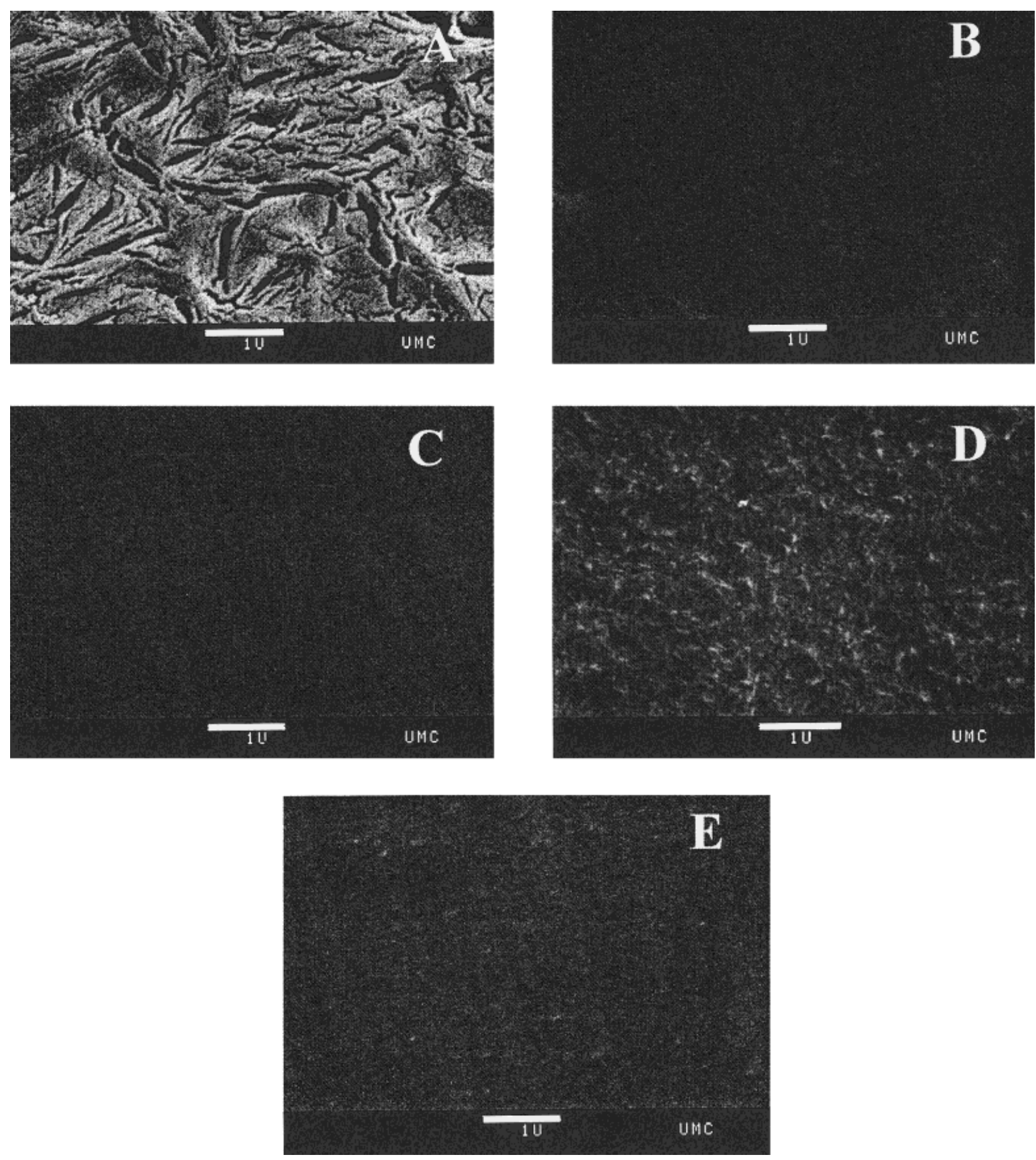

Figure 2. SEM micrographs of untreated (A) POM, (B) PMMA, (C) PET, (D) LDPE, and (E) SR.

structures are generally the most susceptible to $\mathrm{O}_{2}$-plasma degradation, ${ }^{11-13}$ particularly those connected to aliphatic chains. Aromatic polymers are generally more resistant to plasma oxidation and consequently to etching; this characteristic has been attributed to the stabi- lizing effects of the benzene ring. ${ }^{13}$ Siloxanebased materials are probably the least susceptible to degradation from $\mathrm{O}_{2}$-plasma exposure but are greatly susceptible to degradation from $\mathrm{CF}_{4}$ plasmas because of the generation of highly volatile $\mathrm{SiF}_{4}$ products. 

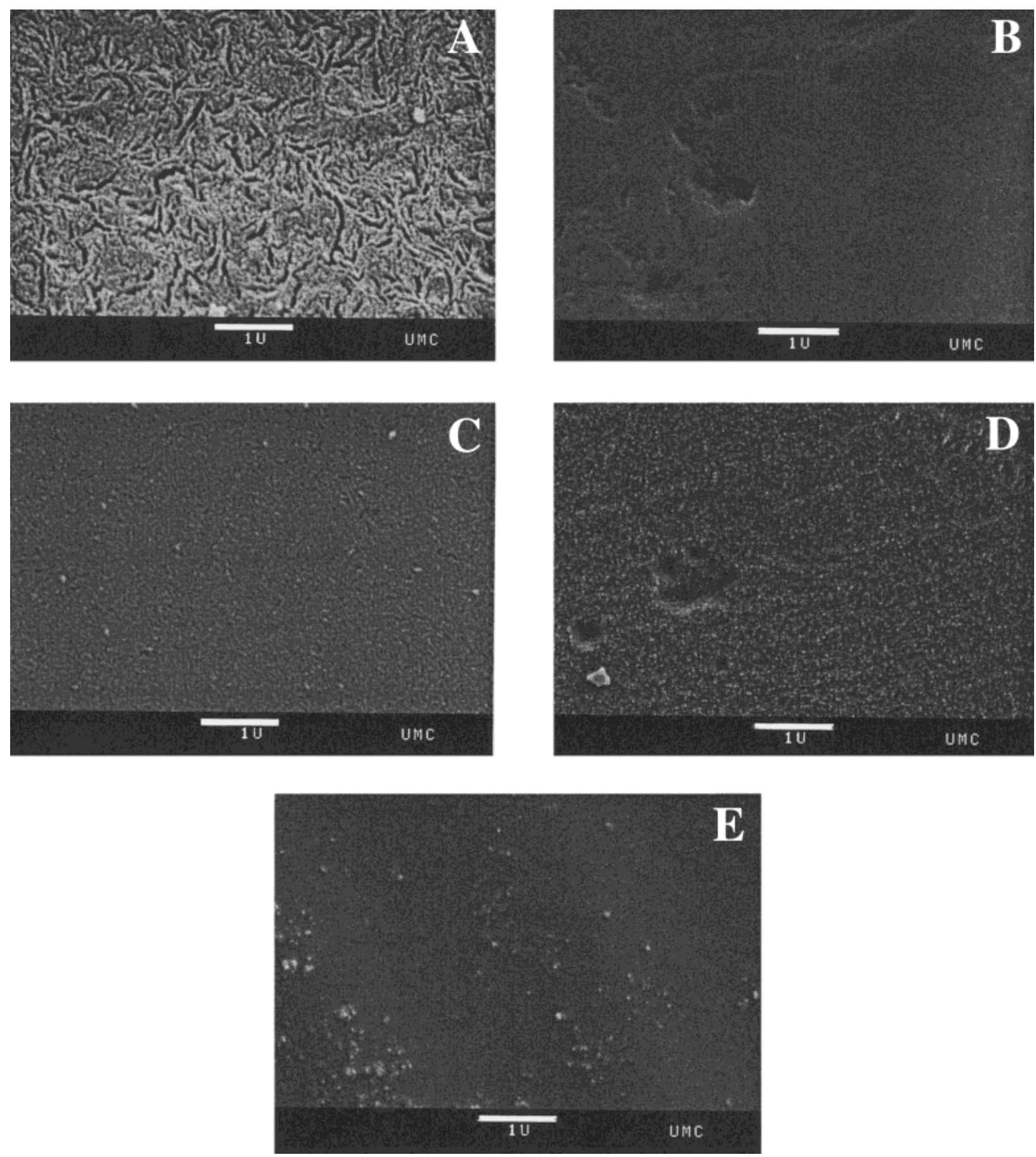

Figure 3. SEM micrographs of $\mathrm{O}_{2}$-plasma-treated (A) POM, (B) PMMA, (C) PET, (D) LDPE, and (E) SR. System pressure, input power, flow rate, and plasma exposure time were fixed at $50 \mathrm{mTorr}, 36 \mathrm{~W}, 1 \mathrm{sccm}$, and $4 \mathrm{~min}$, respectively.

These results regarding the susceptibility of five conventional polymers to oxygen and watervapor plasmas, depicted in Figure 1(A,B), respectively, support the previously discussed trends. Both plasmas had the same effect on the susceptibility of the tested polymers to weight loss; that is, POM $>$ PMMA $>$ PET $>$ LDPE $>$ SR. Thus, POM exhibited the most weight loss, and SR exhibited the least. However, weight loss increased linearly throughout $\mathrm{O}_{2}$ - 

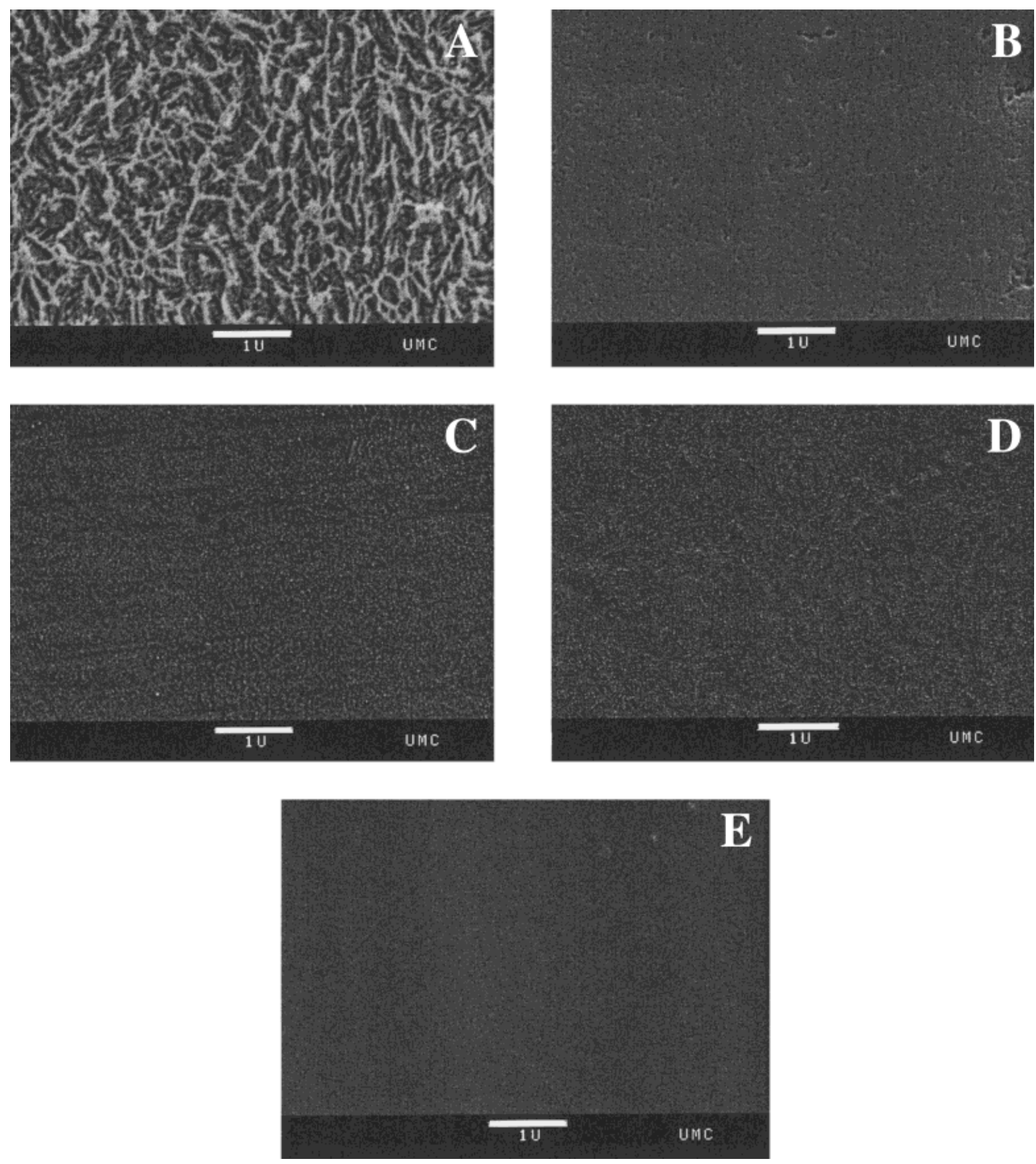

Figure 4. SEM micrographs of $\mathrm{H}_{2} \mathrm{O}$-vapor-plasma-treated (A) POM, (B) PMMA, (C) PET, (D) LDPE, and (E) SR. System pressure, input power, flow rate, and plasma exposure time were fixed at $50 \mathrm{mTorr}, 36 \mathrm{~W}, 1 \mathrm{sccm}$, and $4 \mathrm{~min}$, respectively.

plasma exposure time but only up to about 1 $\min$ of $\mathrm{H}_{2} \mathrm{O}$-vapor exposure time. This indicates $\mathrm{O}_{2}$ plasma continually removes material at a constant rate, whereas $\mathrm{H}_{2} \mathrm{O}$-vapor plasma does so up to a threshold and then gradually begins to taper off until it nearly ceases. However, the weight loss and the etching rates of $\mathrm{H}_{2} \mathrm{O}$-vapor plasma on POM, PMMA, and PET are significantly higher in the first $2 \mathrm{~min}$ in comparison with the oxygen plasma. 

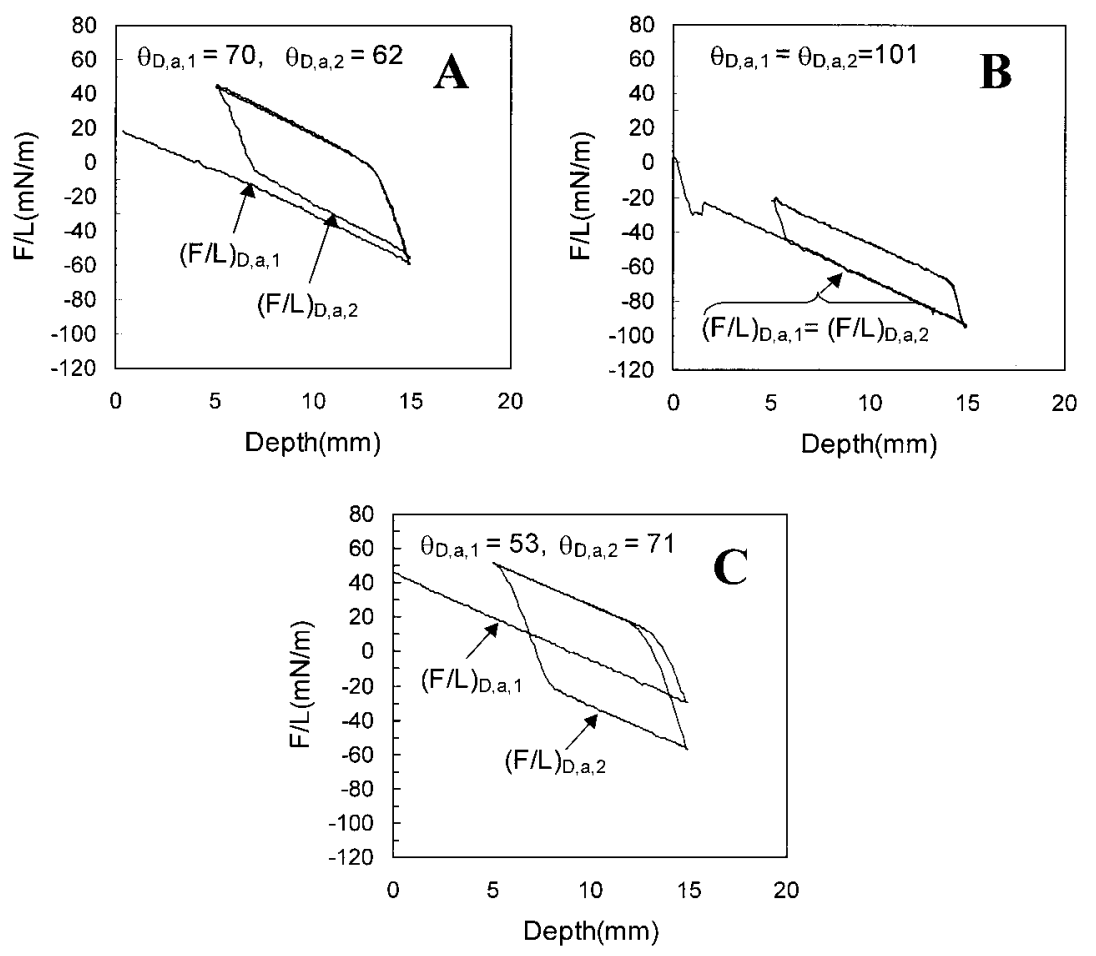

Figure 5. Wilhelmy force loops of (A) untreated nylon-6, (B) untreated LDPE, and (C) $\mathrm{O}_{2}$-plasma-treated LDPE. The second advancing immersion line, $(F / L)_{D, a, 2}$, overlaps the first advancing immersion line, $(F / L)_{D, a, 1}$, for the untreated LDPE but overshoots $(F / L)_{D, a, 1}$ for the $\mathrm{O}_{2}$-plasma-treated LDPE.

The morphology of the untreated polymers in this study, displayed in Figure 2, was rather smooth, with the exception of POM, which exhibited some surface texture and susceptibility to the electron beam, visible in resulting cracking patterns. This is not surprising because POM is known to be susceptible to thermal oxidation and photodegradation. Both plasmas resulted in a slight increase in surface roughness, as is evident in Figures 3 and 4, with the exception of SR, which appeared virtually unaltered at a magnification of 20,000. Plasma-treated POM was much less susceptible to cracking, especially in the case of $\mathrm{H}_{2} \mathrm{O}$-vapor-plasma treatment.

According to $\mathrm{Wu},{ }^{19}$ roughness below 0.5-0.1 $\mu \mathrm{m}$ has a negligible effect on contact-angle measurement. Therefore, the roughness on the plasma-treated samples, with the beam-induced cracking on POM ignored, had a negligible effect on contact-angle measurement.

\section{Surface Oligomers and Wilhelmy Force Loops}

Most relatively unperturbable conventional polymers exhibit Wilhelmy force loops that are paral- lelogram-shaped but vary in size and vertical position depending on three factors: wettability, meniscus shape change, and surface configuration change. ${ }^{7}$ However, many conventional and plasma-modified polymers are perturbable by changing environments. Consequently, those polymers exhibit deviations from the ideal parallelogramshaped force loop that are mainly attributed to surface configuration change. The extent of surface configuration change has been characterized by the measurement of the extent of intrinsic hysteresis from a Wilhelmy force loop. ${ }^{7,20,21}$

Intrinsic hysteresis is the difference between the first and second immersion lines in a Wilhelmy force loop. For many polymeric surfaces, the second immersion line, $(F / L)_{D, a, 2}$, is elevated above the first immersion line, $(F / L)_{D, a, 1}$, because of surface configuration change. This results in an increase of surface hydrophilicity, as indicated by a decrease in the advancing contact angle calculated from the second immersion line, $\theta_{D, a, 2}$, compared to that calculated from the first, $\theta_{D, a, 2}$, depicted in Figure 5(A) for nylon-6.

The thermodynamic driving force for surface configuration change is the local minimization of 

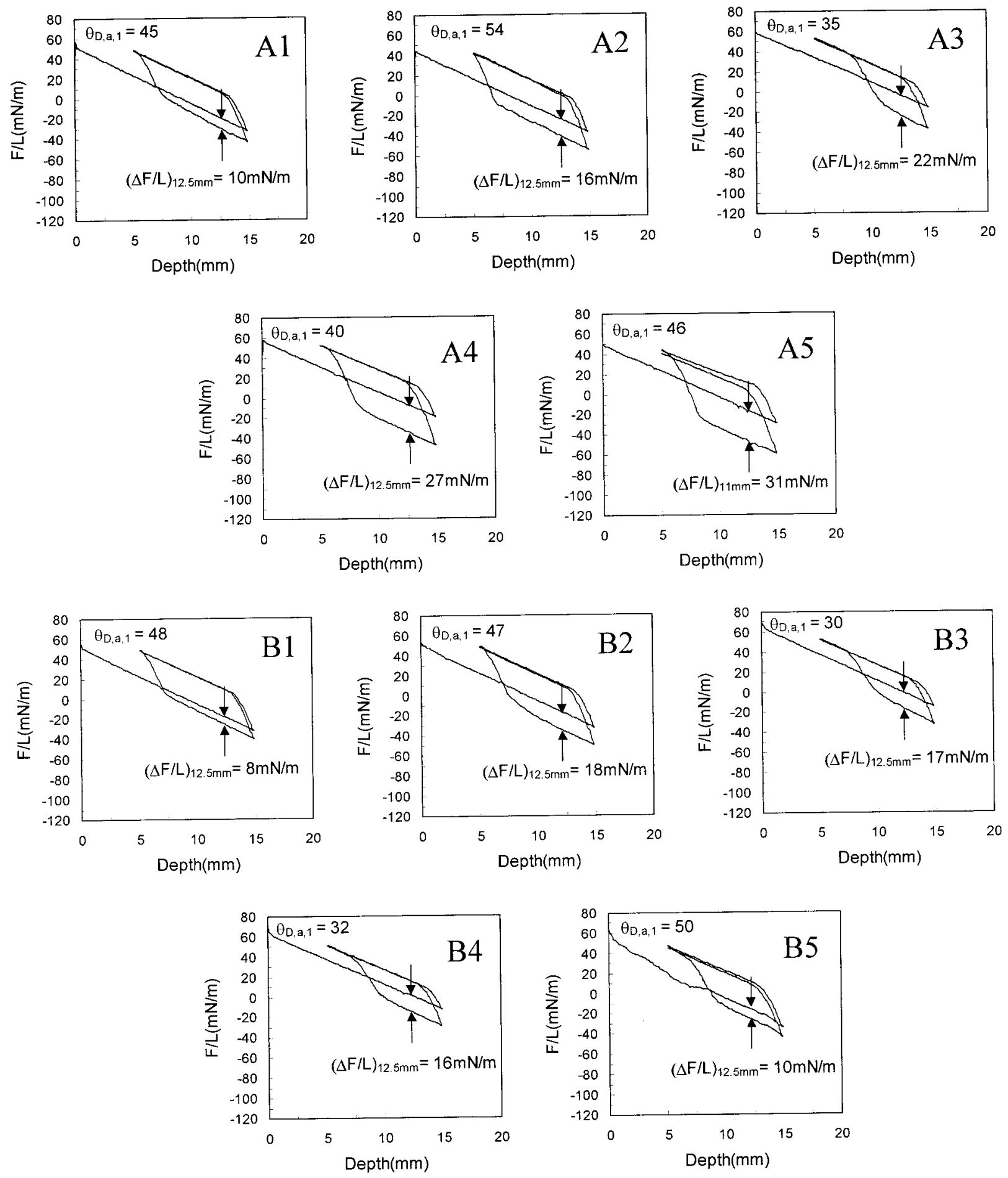

Figure 6. Effect of (A) $\mathrm{O}_{2}$ plasma and (B) water-vapor plasma on Wilhelmy force-loop overshooting [i.e., $(\Delta F / L)_{12.5} \mathrm{~mm}$ ] of (1) POM, (2) PMMA, (3) PET, (4) LDPE, and (5) SR. Plasma discharge conditions were as follows: $1 \mathrm{sccm}, 50 \mathrm{mTorr}, 36 \mathrm{~W}$, and $0.2 \mathrm{~min}$.

interfacial tension induced by wetting with water. Polymers exhibiting intrinsic hysteresis are considered more perturbable to wetting with water in comparison with unperturbable polymer surfaces. Intrinsic hysteresis is absent for unperturbable polymers, as indicated by the retracing of the first 


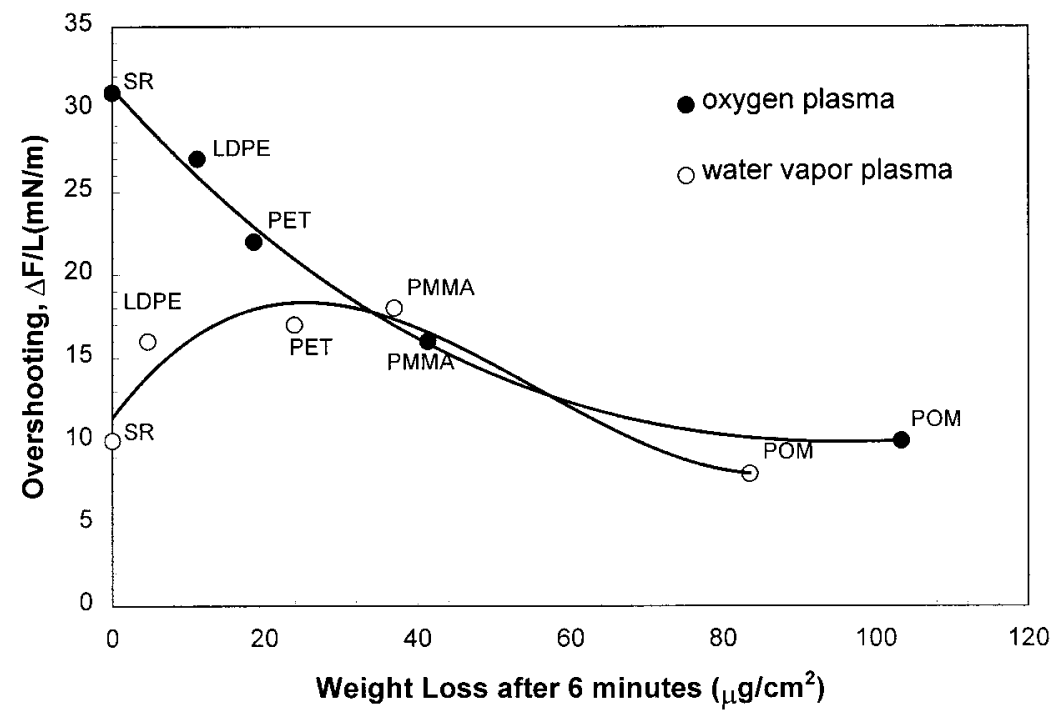

Figure 7. Susceptibility of five conventional polymers to overshooting and weight loss from exposure to $\mathrm{O}_{2}$ and $\mathrm{H}_{2} \mathrm{O}$-vapor plasmas.

and second immersion lines, respectively. An example of this occurrence for untreated LDPE is depicted in Figure 5(B). This is typical force-loop behavior for many conventional polymers, including ultrahigh molecular weight polyethylene, polypropylene, polycarbonate, PET, and poly(vinylidene fluoride). ${ }^{7}$

Current investigations aimed at improving the wettability of LDPE via oxygen and water-vaporplasma treatment have yielded peculiar Wilhelmy force-loop behavior. The second immersion line overshot, or fell below, the first immersion line, as depicted in Figure 5(C). This behavior is not justifiable by thermodynamic surface equilibria, which always tend toward minimization of interfacial tension with contacting water. A perturbable polymer capable of minimizing interfacial tension with water by surface configuration change is indicated by the movement of the second immersion line above the first immersion line. Thus, surface configuration change cannot possibly be the cause of this contradictory behavior.

Overshooting of immersion lines may be understood through a reconsideration of possible residual products from the etching process. Because both $\mathrm{O}_{2}$ and $\mathrm{H}_{2} \mathrm{O}$-vapor plasmas etch volatile products from the surface of polymers, it is also plausible that nonvolatile hydrophilic oligomers are formed because of chain scission and the unzipping of the polymer backbone. These hydrophilic oligomers are only loosely affixed to the surface and thus provide an unstable surface state.

During the first immersion (i.e., advancing), the three-phase contact line moves over the surface laden with oxidized oligomers, which are perceived as part of the surface. However, because the oligomers are only loosely affixed to the surface, they are easily washed away by the polar water during the first emersion (i.e., receding). This exposes a less oxidized or more hydrophobic surface state that is subsequently rewetted during the second immersion. Hydrophobic recovery is evident upon calculation of the first and second immersion advancing contact angles, which correspond to $53^{\circ}$ and $71^{\circ}$, respectively, in Figure 5(C).

The effect of oxygen and water-vapor plasmas on overshooting, indicated by $\Delta F / L$ (i.e., the difference between the first and second immersion lines when the second lies below the first), is depicted in Figure 6. The polymers most susceptible to weight-loss degradation from $\mathrm{O}_{2}$-plasma exposure exhibited the least amount of overshooting. Conversely, polymers less susceptible to weight-loss degradation from $\mathrm{O}_{2}$-plasma exposure exhibited greater overshooting, as depicted in Figure 7. Thus, polymers most susceptible to weight-loss degradation, such as POM, produce more volatile products than nonvolatile oligomers. Polymers less susceptible to weight-loss degradation by chemical etching, such as SR, favor the production of nonvolatile oligomers that 

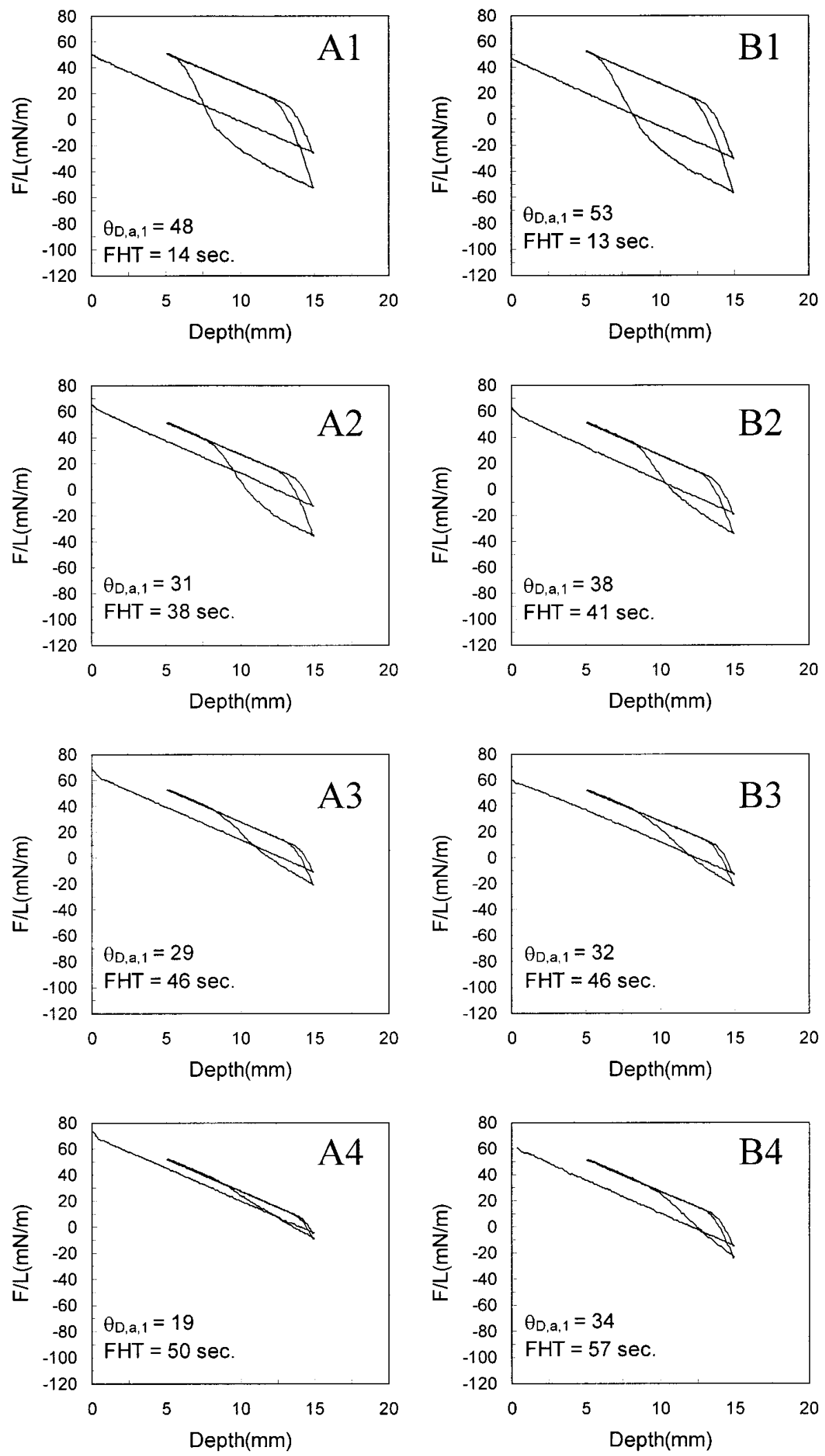

Figure 8. Effect of the $\mathrm{O}_{2}$ flow rate at (A) $1 \mathrm{sccm}$ and (B) $10 \mathrm{sccm}$ and plasma exposure times of (1) $0.2 \mathrm{~min}$, (2) $1 \mathrm{~min}$, (3) $2 \mathrm{~min}$, and (4) min on LDPE Wilhelmy force loops. System pressure and input power were fixed at $50 \mathrm{~m}$ Torr and $36 \mathrm{~W}$, respectively. The dynamic advancing contact angle, $\theta_{D, a, 1}$, and FHT are shown in each plot. 

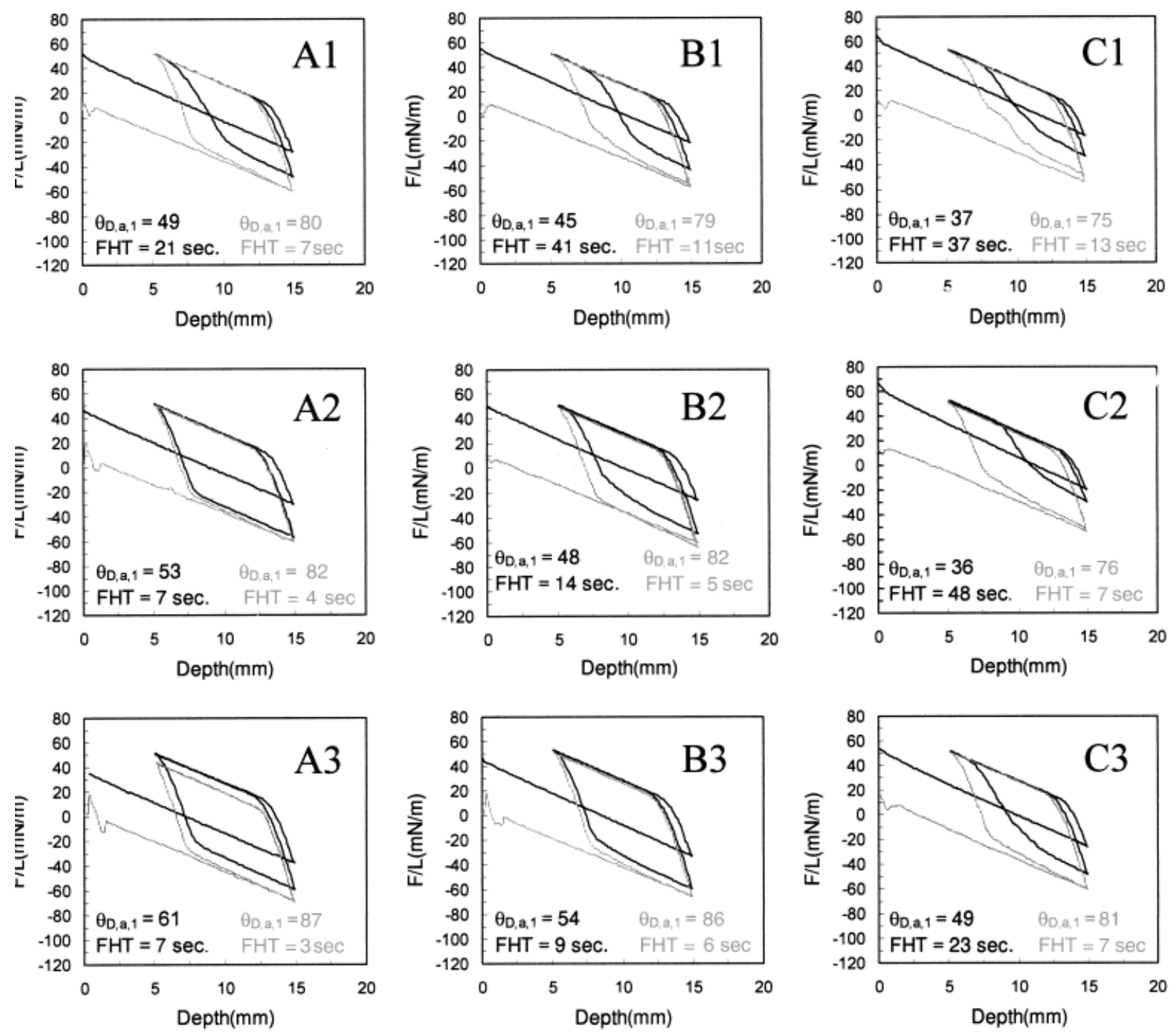

Figure 9. Effects of input power at (A) $8 \mathrm{~W}$, (B) $30 \mathrm{~W}$, and (C) $63 \mathrm{~W}$ and system pressures of (1) 25 mTorr, (2) 50 mTorr, and (3) 100 mTorr on Wilhelmy force loops of $\mathrm{O}_{2}$-plasma-modified LDPE. Dark force loops were measured just after samples were removed from the reactor; gray force loops were measure 2 weeks later after equilibration with air.

stay stagnant on the surface. $\mathrm{H}_{2} \mathrm{O}$-vapor plasma has a similar effect on polymers more susceptible to chemical etching. However, $\mathrm{H}_{2} \mathrm{O}$-vapor plasma suppresses the production of surface oligomers. Thus, less $\mathrm{H}_{2} \mathrm{O}$-vapor-plasma-induced overshooting than $\mathrm{O}_{2}$-plasma-induced overshooting is evident for polymers least susceptible to etching (Fig. 7).

Short-term hydrophobic recovery resulting from the production of surface oligomers, which occurs within the timescale of wetting during Wilhelmy force measurements, should be distin- guished from long-term hydrophobic recovery resulting from surface configuration change, which occurs over a period of days, weeks, or even months under ambient air conditions. Short-term hydrophobic recovery is permanent, whereas long-term hydrophobic recovery may be at least partially reversed via immersion in water. The irreversible effects of short-term hydrophobic recovery are detrimental to wetting stability and, therefore, must be minimized. This necessity prompted a detailed look at the effects of plasma discharge conditions on wetting stability and flu- 

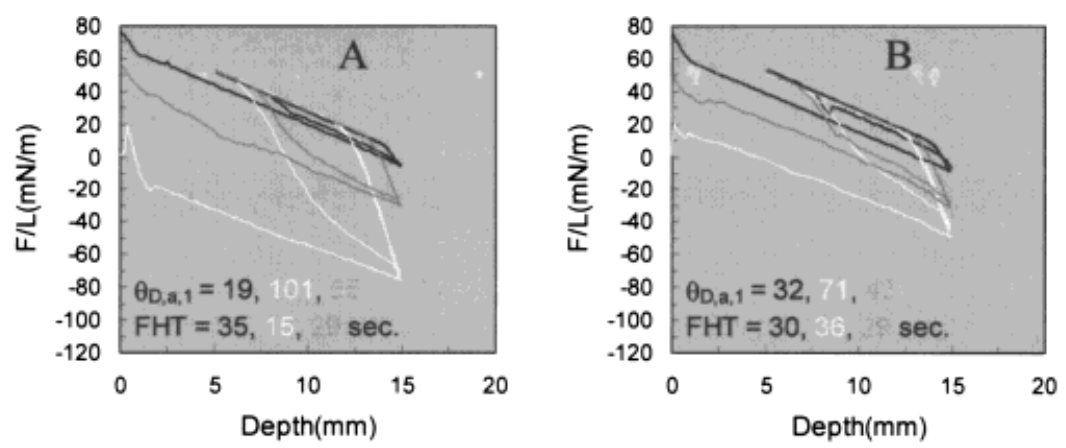

Figure 10. Effect of (A) oxygen plasma and (B) water-vapor plasma on LDPE Wilhelmy force loops. Dark force loops were figured immediately after samples were removed from the reactor. White force loops were after the same samples were exposed to ambient air for $24 \mathrm{~h}$. System pressure, input power, flow rate, and plasma exposure time were fixed at $25 \mathrm{mTorr}, 60 \mathrm{~W}, 10 \mathrm{sccm}$, and $4 \mathrm{~min}$, respectively.

id-holding capability on $\mathrm{O}_{2}$ - and $\mathrm{H}_{2} \mathrm{O}$-vapor-plasma-modified LDPE.

\section{Effect of Discharge Conditions on Wetting and Fluid-Holding Capability}

The FHT parameter was used to semiquantitatively characterize the capability of polymeric surfaces to hold a continuous film of water. More specifically, FHT measures the time it takes a continuous film of water to recede down the side of a Wilhelmy plate. In general, high wettability is the most favorable condition for continuous water films, although some hydrophobic surfaces are possible if they are perturbable by water. ${ }^{17}$ If a continuous water film indeed adheres to the surface, it is reflected in the corresponding Wilhelmy force loop by the second immersion line retracing the first emersion line. This results in a hookshaped force loop rather than the typical parallelogram-shaped force loop. The FHT is calculated from the length of the retraced region and the speed of immersion, which is discussed in more detail elsewhere. ${ }^{17}$

Wettability, indicated by the first immersion advancing contact angle, $\theta_{D, a, 1}$, and the fluidholding capability, indicated by the FHT, varied profoundly for plasma-modified LDPE, depending on the glow discharge conditions. The effects of $\mathrm{O}_{2}$-plasma exposure time and inlet gas flow rate on $\theta_{D, a, 1}$ and FHT are depicted in Figure 8 . The most obvious result from both $\mathrm{O}_{2}$ and $\mathrm{H}_{2} \mathrm{O}$-vapor plasmas is that wettability and FHT increased with increasing plasma exposure time. However, the most important result of increasing exposure time to both plasmas is the reduction in over- shooting. Surface oligomers generated early on are subsequently removed by prolonged exposure. Oxidation of the chemically attached surface prevails over oligomer production after extended periods of exposure to plasma.

The effects of system pressure and input power on wettability and FHT immediately following exposure to $\mathrm{O}_{2}$ plasmas are depicted as the dark lines in Figure 9. Wettability and FHT changed very little with system pressure in comparison to input power for both plasmas. In general, for both $\mathrm{O}_{2}$ and $\mathrm{H}_{2} \mathrm{O}$-vapor plasmas, lower pressure and higher input power were the best conditions under which to maximize wettability and minimize overshooting. However, overshooting was generally much smaller after exposure to $\mathrm{H}_{2} \mathrm{O}$-vapor plasma than after exposure to $\mathrm{O}_{2}$ plasma.

After 2 weeks of equilibration with ambient air, the samples were rewetted, as indicated by the gray lines in Figure 9. Further hydrophobic recovery resulted, but this time it was due to surface configuration change. FHT was greatly diminished because oxidized oligomers were washed away during the first Wilhelmy force measurement.

Based on the latter results, the following discharge conditions were chosen to maximize wettability and minimize overshooting: $4 \mathrm{~min}, 10$ sccm, 25mTorr, and $63 \mathrm{~W}$. The results depicted in Figure 10 show that overshooting was indeed eliminated and high wettability as well as fluidholding capability was achieved. However, the high wettability was short-lived after exposure to ambient air for 2 weeks because of the perturbability of the surface to air. The $\mathrm{O}_{2}$-plasmatreated LDPE recovered its hydrophobicity completely, whereas the $\mathrm{H}_{2} \mathrm{O}$-vapor-plasma-treated 

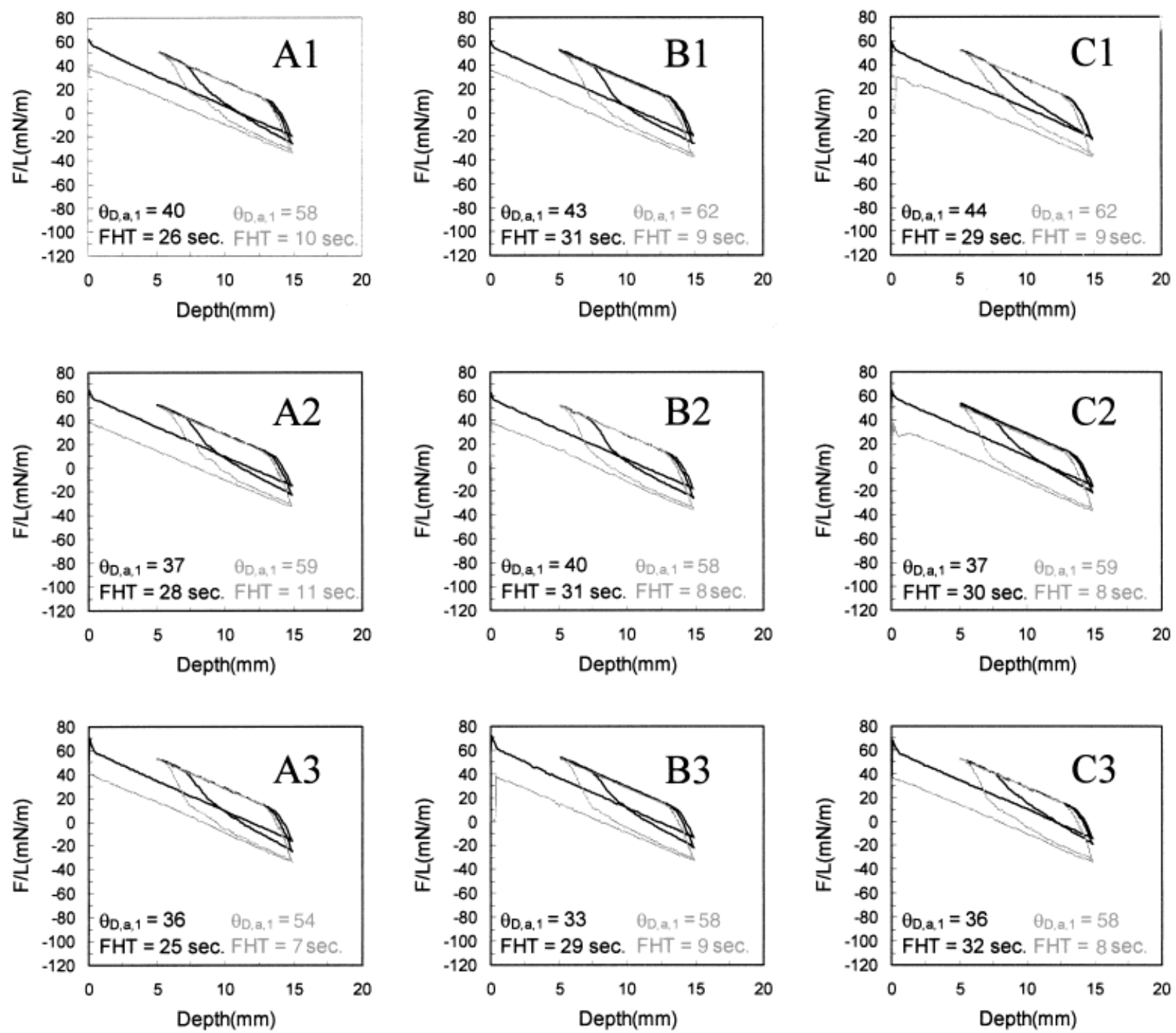

Figure 11. Effects of post-oxygen-plasma-treatment at system pressures of (A) 25 mTorr, (B) 50 mTorr, and (C) 100 mTorr and input powers of (1) $8 \mathrm{~W}$, (2) $30 \mathrm{~W}$, and (3) $60 \mathrm{~W}$ on $\mathrm{CH}_{4}$ /air-plasma-polymer-coated LDPE. The $\mathrm{O}_{2}$ flow rate and exposure time were fixed at $10 \mathrm{sccm}$ and $0.2 \mathrm{~min}$, respectively. Discharge conditions for $\mathrm{CH}_{4} /$ air deposition were 2/1 sccm, $50 \mathrm{mTorr}, 36 \mathrm{~W}$, and $10 \mathrm{~min}$. Dark force loops were measured immediately after samples were removed from the reactor; gray force loops were measured 2 weeks later after equilibration with ambient air.

LDPE was far more resilient. Soaking the same samples in DDI water for $24 \mathrm{~h}$ regained any lost fluid-holding capability and surface hydrophilicity, but more so for $\mathrm{H}_{2} \mathrm{O}$-vapor-plasma-treated LDPE.

There are a number of factors that influence the extent and rate of hydrophobic recovery, including environment, temperature, crystallinity, and crosslinking. However, several investigators have shown that crosslinking is a very effective means of restricting the mobility of hydrophilic and hydro- phobic moieties to and from the surface. ${ }^{4,9}$ Yasuda et al. ${ }^{4}$ showed that depositing a thin layer of plasma polymer before polypropylene was exposed to post$\mathrm{O}_{2}$-plasma restricted hydrophobic recovery completely after 30 days in ambient air. Polypropylene exposed only to $\mathrm{O}_{2}$ plasma recovered completely within 20 days. Many plasma polymer films are highly crosslinked, that is, every $2-4$ carbons as opposed to every 100-1000 carbons in many conventional polymers. This severely restricts the mobility of hydrophilic functional groups introduced by 
the post- $\mathrm{O}_{2}$-plasma-treatment and thus limits surface configuration change.

The effects of depositing a $\mathrm{CH}_{4} /$ air-plasma polymer prior to exposure to $\mathrm{O}_{2}$ plasma on hydrophobic recovery are displayed in Figure 11. The same $\mathrm{O}_{2}$-plasma discharge conditions used in Figure 9 were employed. Hydrophobic recovery from both surface oligomers and surface configuration change was restricted. Thus, the plasma polymer layer not only restricts the mobility of hydrophilic functional groups but also retards the production of oxidized oligomers on the surface. Furthermore, both wettability and FHT behave virtually independent of pressure and input power in the case of $\mathrm{O}_{2}$-plasma exposure.

\section{CONCLUSIONS}

The presence of unstable surface oligomers was indicated when the second immersion line overshot, or fell below, the first immersion line in a Wilhelmy force loop within the timescale of wetting. Long-term hydrophobic recovery resulting from oxidized oligomers was irreversible, whereas short-term hydrophobic recovery resulting from surface configuration change could be reversed via immersion in water. This study distinguished the two by identifying differences in the shapes of the corresponding Wilhelmy force loops and in the fluid-holding parameter. The presence of surface oligomers was most detrimental to wetting stability and fluid-holding capability but could be controlled via the type of reactive gas, the discharge conditions, and the polymer substrate.

Polymers most susceptible to weight-loss degradation from $\mathrm{O}_{2}$-plasma and $\mathrm{H}_{2} \mathrm{O}$-vapor-plasma exposure, such as POM, exhibited the least amount of Wilhelmy overshooting. Thus, for these polymers, the production of volatile products is favored over that of nonvolatile oligomer products. Polymers less susceptible to weight-loss degradation from exposure to $\mathrm{O}_{2}$ plasma showed correspondingly increased overshooting. Thus, for such polymers, the production of nonvolatile oligomer products is favored over that of volatile products.

In general, exposure to $\mathrm{H}_{2} \mathrm{O}$-vapor plasma had an important advantage over exposure to $\mathrm{O}_{2}$ plasma: it consistently lessened hydrophobic recovery (evident in the reduced overshooting) for those polymers least susceptible to weight loss. Discharge conditions were manipulated to maximize wettability and fluid-holding capability while minimizing overshooting: longer exposure time and higher discharge power had the greatest impact. The benefits of crosslinking were utilized to minimize oligomer production by a layer of methane/air-plasma polymer being deposited before exposure to a reactive plasma. This significantly reduced hydrophobic recovery from both surface oligomers and surface configuration change.

The SEM micrographs, taken by Lou Ross at the Geological Sciences Department of the University of Missouri-Columbia, were greatly appreciated.

\section{REFERENCES AND NOTES}

1. Yasuda, H.; Charlson, E. J.; Charlson, E. M.; Yasuda, T.; Miyama, M.; Okuno, T. Langmuir 1991, 7, 2394-2400.

2. Drost-Hansen, W. Ind Eng Chem 1969, 61, 10-47.

3. Yasuda, T.; Okuno, T.; Yasuda, H. Langmuir 1994, 10, 2435-2439.

4. Yasuda, H.; Sharma, A. K.; Yasuda, T. J Polym Sci A 1981, 19, 1285-1291.

5. Yasuda, T.; Okuno, T.; Ysuji, K.; Yasuda, H. Langmuir 1996, 12, 1391-1394.

6. Yasuda, T.; Miyama, M.; Yasuda, H. Langmuir 1992, 8, 1425-1430.

7. Weikart, C. M.; Miyama, M.; Yasuda, H. K. J Colloid Interface Sci 1998, 211, 28-38.

8. Morra, M.; Occiello, E.; Garbassi, F. J Adhes Sci Technol 1973, 7, 1051-1063.

9. Van Der Mei, H. C.; Stokroos, J. M.; Schakenraad, J. M.; Busscher, H. J. J Adhes Sci Technol 1991, 9, 757-769.

10. Chapiro, A. Radiation Chemistry of Polymeric Systems; Wiley: New York, 1962; pp 1-75.

11. Yasuda, T.; Gazicki, M.; Yasuda, H. J Appl Polym Sci 1984, 38, 201-214.

12. Yasuda, H.; Lamaze, C. E.; Sakaoku, K. J Appl Polym Sci 1973, 17, 137-152.

13. Moss, S. J. Polym Degrad Stab 1987, 17, 205-222.

14. Hansen, R. H.; Pascale, J. V.; De Benedictis, T.; Rentzepis, P. M. J Polym Sci A 1965, 3, 2205-2214.

15. Inagaki, N.; Tasaka, S.; Horiuchi, T.; Suyama, R. J Appl Polym Sci 1998, 68, 271-279.

16. Poncin-Epaillard, F.; Chevet, B.; Brosse, J.-C. Eur Polym J 1990, 26, 333-339.

17. Weikart, C. M.; Miyama, M.; Yasuda, H. K. Langmuir, submitted for publication, 1999.

18. Weikart, C. M.; Miyama, M.; Yasuda, H. K. J Colloid Interface Sci 1998, 211, 18-27.

19. Wu, S. Polymer Interface and Adhesion; Marcel Dekker: New York, 1982; pp 15-26.

20. Miyama, M.; Yang, Y.; Yasuda, T.; Okuno, T.; Yasuda, H. Langmuir 1997, 13, 5494-5503.

21. Miyama, M.; Yasuda, H. K. Langmuir 1998, 14, 960-964. 